\title{
Life-span changes in implicit and explicit memory
}

\author{
PETER GRAF \\ University of British Columbia, Vancouver, British Columbia, Canada
}

\begin{abstract}
The literature shows that whereas explicit memory decreases in later life, implicit memory tends to remain intact. This pattern of results is mirrored in the findings from recent experiments with young children who show age-related increases in explicit but not implicit memory. This paper summarizes research on lifespan changes in implicit and explicit memory. The findings are discussed in terms of broad theoretical accounts that have been used to explain, on the one hand, dissociations between implicit and explicit memory in healthy adults and in memorydisordered patients, and, on the other hand, the development of memory in early childhood.
\end{abstract}

Most investigations of implicit and explicit memory have concentrated on three broadly defined areas. First, studies with college students have shown that a wide range of experimental manipulations have different effects on performance of implicit and explicit memory tests. Second, extensive research with organic amnesic patients has revealed that implicit memory test performance can be entirely normal even when explicit remembering is severely impaired. A third line of investigation has focused on memory changes in later life; this work suggests that even though explicit remembering tends to decline in late adulthood, aging has no effect or has only a minimal effect on implicit memory. Recently, the latter research has been supplemented by studies of implicit and explicit memory in young children. What is emerging from the combined work with adults and young children is a complete picture of life-span changes in implicit and explicit memory. In the first part of this paper, I briefly sketch this picture; in the second part, I will describe three kinds of interpretations that have been offered to explain it.

\section{Review of Research}

Aging. The review begins with studies of memory changes in later life. The typical study in this area has two subject groups: adults around 70 years of age, and college students around 20 years of age. Every experiment has a learning phase during which some materials are presented for study, and, after a variable delay, both implicit and explicit memory tests are given. What differs between experiments are the study tasks and the study materials, the latter of which consist of words, pairs of words, or line drawings of common objects. The experiments differ also in how implicit memory was assessed, and I have used the different tests to organize this review.

This paper was presented at the 30th annual meeting of the Psychonomic Society in Atlanta, November 1989. Send requests for reprints to Peter Graf, Department of Psychology, University of British Columbia, Vancouver, BC V6T 1Y7, Canada, or via e-mail to graf@ubcmtsg.bitnet.CA.
This paper offers no new findings on explicit memory; it is well known that explicit remembering declines in late adulthood, and the extent of this decline is larger on free and cued recall tests than on recognition tests. A comprehensive summary of age-related changes in explicit remembering is provided in Salthouse's (1982) book Adult Cognition, as well as in several other books, chapters, and review papers (e.g., Kausler, 1982).

In contrast to explicit remembering, which can show age-related decreases of $50 \%$ or more, the recent literature suggests that aging has a less or no detrimental effect on performance on implicit memory tests. What is the evidence for this claim? The findings from the extant studies show considerable variability. In order to highlight general trends, I have used a method to summarize and display the results that ignores differences between experiments and focuses attention on the overall amount of priming shown by subjects from different age groups. Different tests provide different indexes of priming, and the results are thus shown in a separate figure for each test type.

Figure 1 shows priming on word identification tests. Such tests require subjects to identify words that are presented very briefly, and priming is indicated by facilitated identification of words studied previously. The data are from studies by Light and Singh (1987). There were four different conditions, and despite differences among them, the results highlight the similar amounts of priming in younger and older adults.

The next figure (Figure 2) shows priming on a homophone spelling test. For this test, homophones like deer/dear are spoken and subjects must write them or use them in a sentence. Priming reflects the extent to which a prior encounter with a less common interpretation of the homophone biases its subsequent spelling. The data in Figure 2 come from experiments by Howard (1986, 1988), and by Rose, Yesavage, Hill, and Bower (1986). Two of five conditions show no evidence of an age-related change in priming, and three conditions show a clear difference. Overall, the older adults showed only $69 \%$ 


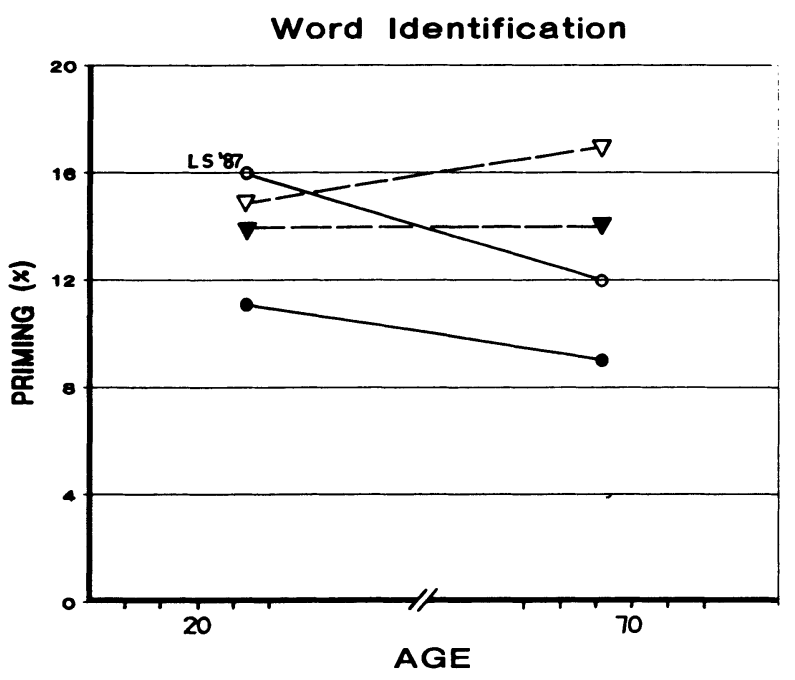

Figure 1. Word identification test priming by younger and older adults. The results are from experiments by Light and Singh (1987).

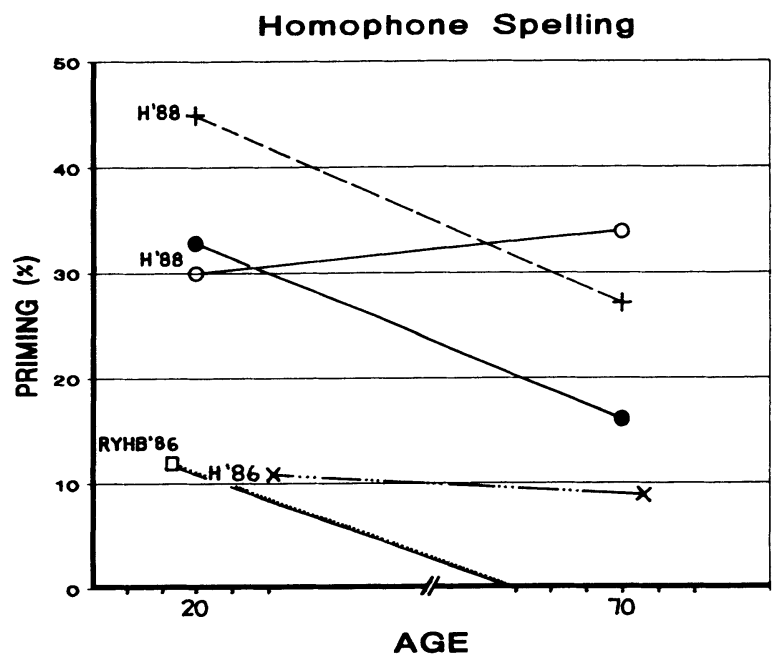

Figure 2. Homophone spelling test priming by younger and older adults. The results are from experiments by Howard (1986, 1988), and by Rose, Yesavage, Hill, and Bower (1986).

(unweighted mean) as much priming as did their younger counterparts.

A similar age-related decrease in the magnitude of priming effects is highlighted by Figure 3. The data are from completion tests, which require subjects to complete word stems or fragments with the first words that come to mind. Priming is revealed by an increase in the probability of completing cues with target words that were previously studied. The figure shows the results from work by Light and colleagues (Light \& Singh, 1987; Light, Singh, \& Capps, 1986). Overall, the older group showed only $74 \%$ as much priming as did the younger subjects. This agerelated decrease occurred in all but one condition-when priming was assessed after a 7-day delay and performance levels were very low.
The next figure (Figure 4) shows completion test effects with paired words-priming for new associations. These findings are particularly relevant, because it is sometimes assumed that older adults have a special problem with new learning. The data come from Howard (1988), and Graf and Schacter (1985). The difference in age between the subject groups in the latter study was small (27 years), and the results show no age-related decline in performance. However, there was a noticeable age-related decrease in priming in Howard's study. Across all conditions shown in the figure, the older groups showed only $84 \%$ as much priming as did their younger counterparts.

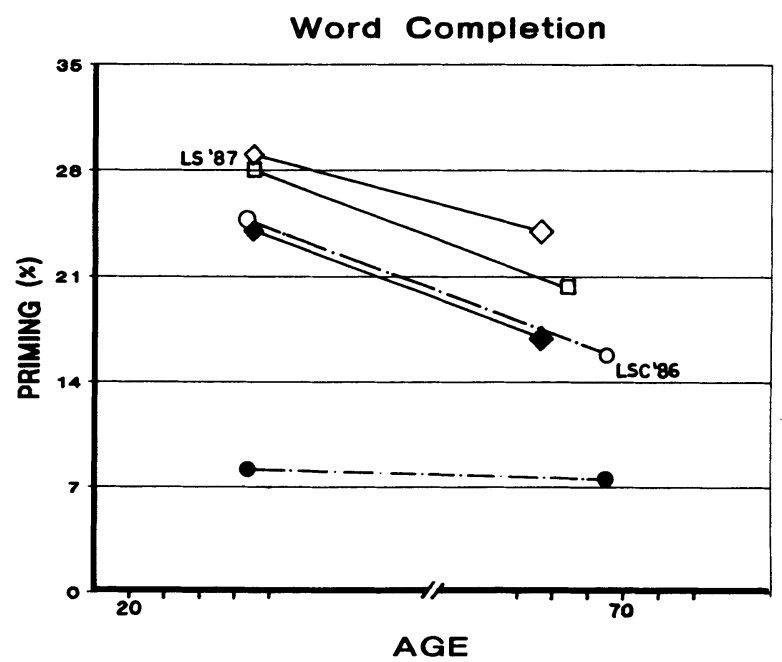

Figure 3. Word completion test priming by younger and older adults. The results are from experiments by Light and Singh (1987), and by Light, Singh, and Capps (1986).

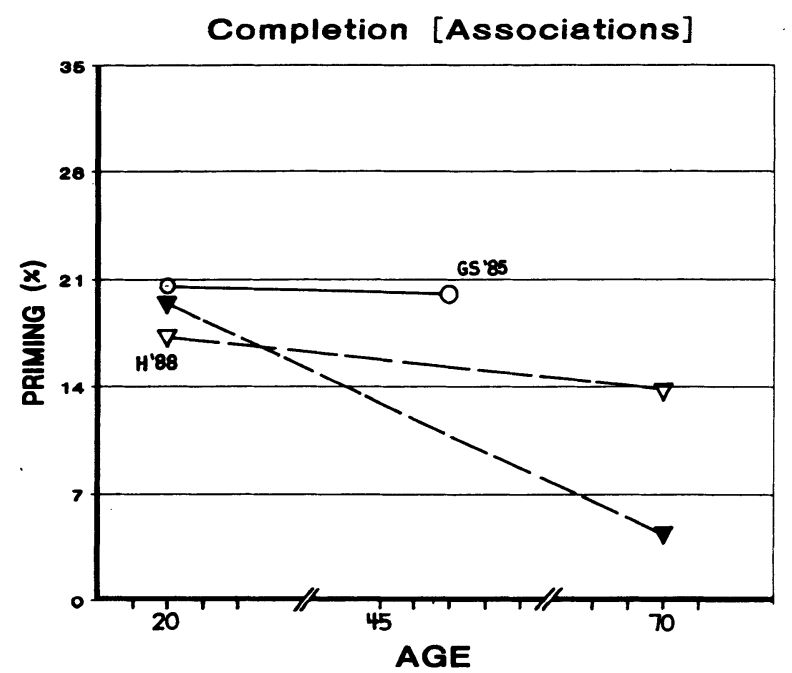

Figure 4. Word completion test priming for newly acquired associations by younger and older adults. The results are from experiments by Graf and Schacter (1985), and by Howard (1988). 
The final figure (Figure 5) shows priming on associative recognition tests. For such tests, subjects are required to make yes/no recognition decisions about individual words that have been studied in pairs. If a word is tested immediately following another that was paired with it at study, decision times tend to be faster, thereby revealing a priming effect. The data in Figure 5 come from Howard and colleagues (Howard, 1988; Howard, Heisey, \& Shaw, 1986) and from Rabinowitz (1986). With two exceptions, the findings show a consistent pattern-similar amounts of priming across age groups.

This brief review is not exhaustive; however, it provides a sufficient basis for speculating about the influences of aging on performance on implicit memory tests. Averaged across all the studies, the older group showed only $82 \%$ (unweighted mean) as much priming as did their younger counterparts. While this reduction in priming should not be ignored and remains to be explained, it must be viewed in proper perspective. A typical experiment may show a $20 \%$ priming effect in young adults, and only a $16 \%$ (i.e., $80 \%$ of $20 \%$ ) effect in older adults, and this difference is minimal when compared with the far greater age-related decreases in explicit remembering, which can reach $50 \%$ or more.

Moreover, there are several reasons to suspect that the extant studies exaggerate age differences in priming. The main reason is a lack of experimental control over what subjects do on priming tests. If subjects figure out that a test is related to a previously studied list, they may change their strategy and engage in explicit remembering. A change in strategy is more likely to occur with younger subjects who remember more of the study list. Previous investigators were aware of this confound, and when they manipulated conditions to prevent or minimize a change in strategy, priming effects were similar across

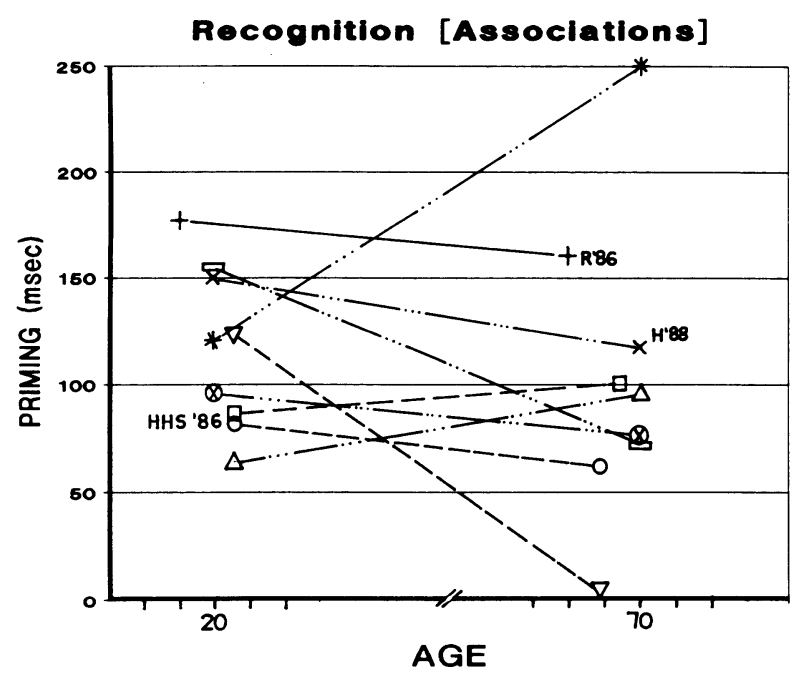

Figure 5. Recognition test priming for newly acquired associations by younger and older adults. The results are from experiments by Rabinowitz (1986), by Howard (1988), and by Howard, Heisey, and Shaw (1986).

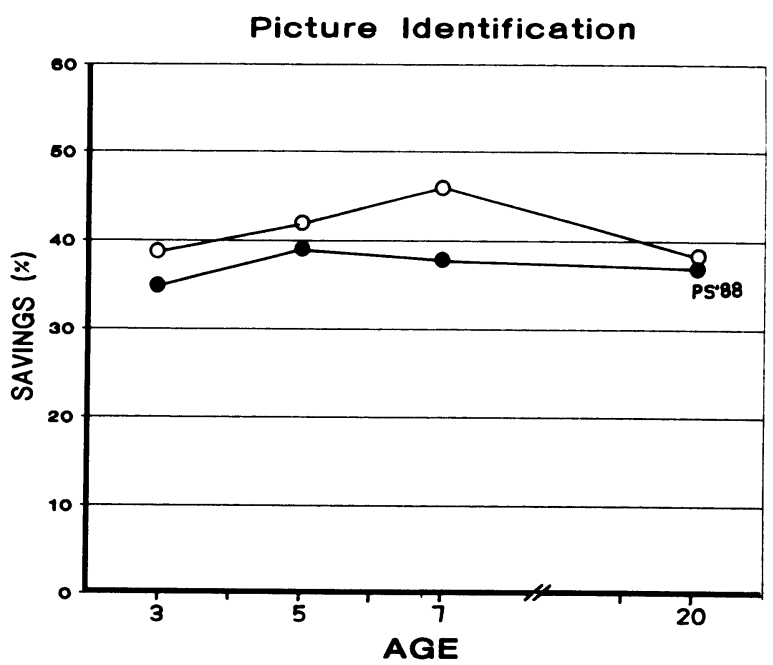

Figure 6. Picture identification test priming by children and young adults. The results are from an experiment by Parkin and Streete (1988).

age groups (see Light et al., 1986). Additional evidence that points to a strategic confound comes from tests such as word identification, which minimize the use of explicit remembering strategies; the results from these tests also show comparable priming effects across age groups. For these reasons, and despite much of the extant data, it seems defensible (for now) to claim that aging has a minimal or no detrimental effect on performance on implicit memory tests.

Development. The following section is a brief review of the extant research on the development of implicit and explicit memory in young children. It offers no new findings on explicit memory, which is well known to develop throughout the early years of life (e.g., Bjorklund \& Bjorklund, 1985). Even though the development of implicit memory in children has been examined in fewer than a handful of studies, their results are consistent and interesting. In one of these studies, Parkin and Streete (1988) assessed priming in 3-, 5-, 7-, and 20-year-olds with a picture identification test. Subjects were required to identify pictures that were degraded to various degrees by deleting a proportion of their line segments. Priming was measured by the subjects' ability to identify a previously seen picture from a more degraded display than at study. The results-expressed as saving scores-are shown in Figure 6. The top line shows priming after a 1-h delay; the bottom line shows priming after a 2-week delay. The figure highlights the enduring nature of priming across delays, and more importantly, the similar priming effects across age groups.

A second study comes from Carroll, Byrne, and Kirsner (1985), who examined picture naming in 5-, 7-, and 9-year-olds. The results, displayed in Figure 7, show the amount of priming following study conditions that required either classifying target pictures (top line) or making decisions about incidental picture details (bottom line). 


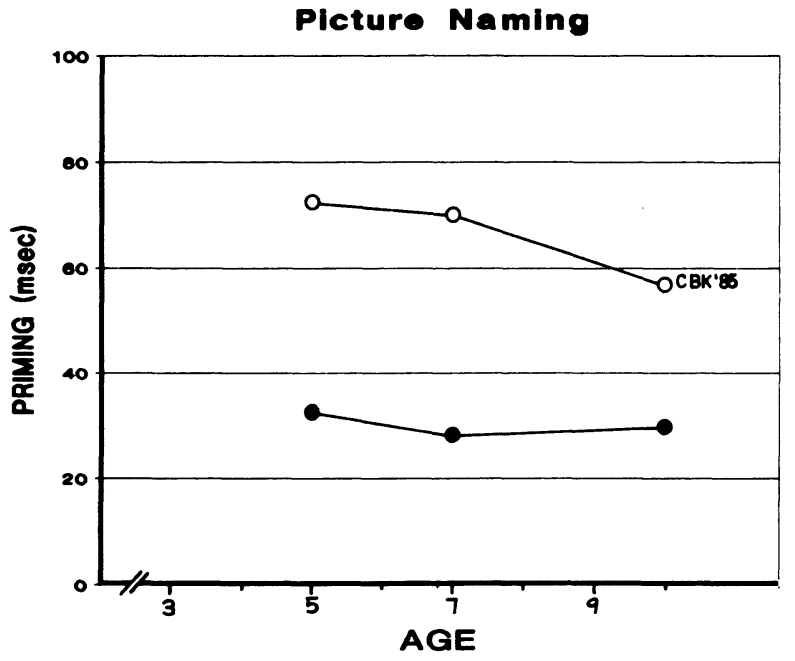

Figure 7. Picture naming test priming by children from different age groups. The results are from experiments by Carroll, Byrne, and Kirsner (1985).

Apart from the large effect due to the study-task manipulation, the figure highlights the similar priming effects across age groups.

Figure 8 shows priming by 3-, 4-, and 5-year-old preschoolers on a category naming test. For this test, subjects are told a category and required to name items from that category; priming is reflected by the increased probability of naming items from a previously studied list. The results are from a study by Greenbaum and Graf (1989), which involved the use of items from child-normed and adult-normed categories. The figure highlights the similar priming effects across age groups with the familiar, child-normed materials, and the age-related increase in priming with the adult-normed materials. A recent study by Terri Buller-a graduate student working with meprovides additional insight into priming in young preschoolers. Buller's study involved a levels-of-processing manipulation, and she found similar priming effects across age groups after the semantic study task $(21 \%, 18 \%$, and $22 \%$, respectively, with 3-, 4-, and 5-year-olds), together with an age-related increase in priming after the nonsemantic study task $(4 \%, 14 \%$, and $12 \%$, respectively, with 3-, 4-, and 5-year-olds).

To my knowledge, this small collection includes all published studies on the development of priming. Two aspects of the findings are noteworthy. First, the results in Figures 7 and 8 show different priming effects across study tasks. This contrasts with evidence from adult studies in which similar manipulations have had a minimal effect or no effect (e.g., Graf \& Mandler, 1984). Future investigations of this age difference will likely provide insight into the early development of priming. The second and more critical finding is the similarity in priming effects across age groups under some experimental conditions. This finding highlights the fact that at least some aspects of implicit memory are already functional even in young preschoolers, thereby contrasting with the well-known finding that explicit memory increases rapidly during the same period of life.

Summary of findings. In combination, the main findings from the studies with adults and children can be summarized as follows: On the one hand, there is growing support for the view that at least some aspects of implicit memory are already functional during the early preschool years, and that they tend to remain intact well into late adulthood. On the other hand, there is a wealth of evidence showing that the capacity for explicit remembering is acquired and develops during the preschool and school-age period, remains relatively stable throughout adulthood, and declines in old age.

\section{Theoretical Views}

A number of theoretical views have been offered to explain some or all of these findings. The following section briefly outlines three such views: a systems view, a process view, and a task analysis view. These views were selected because they represent the different-but complementary-theoretical approaches that guide current investigations of implicit and explicit memory.

Systems view. Systems views postulate the existence of two memory systems-one for implicit memory and one for explicit memory. The literature gives several examples of such views. A developmental version was proposed by Schacter and Moscovitch (1984), who distinguished between an early and a late developing memory system. They based their argument primarily on the finding that infants as young as $\mathbf{8}$ months of age can show evidence of learning on a number of tasks, basically those that involve habituation and conditioning-tasks that do not require explicit remembering. They suggested that performance on these tasks and, by extension, on tests of implicit memory is mediated by the early system, and

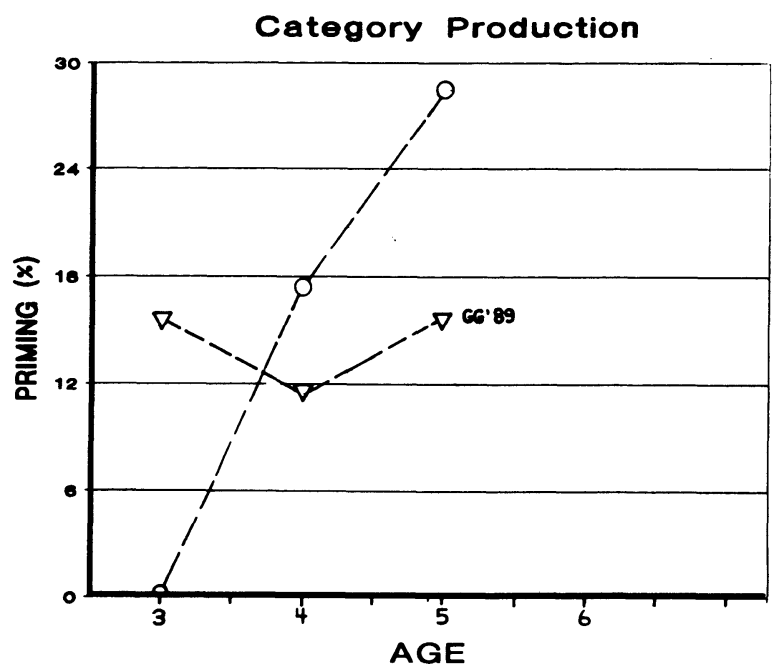

Figure 8. Category production test priming by preschoolers from different age groups. The results are from an experiment by Greenbaum and Graf (1989). 
that the capacity for true or explicit remembering is acquired later in life with the development of the late system. This view can also explain age-related changes in adult memory. It is possible that the late system suffers more from the traumas and strains accumulated in the course of life, and thus, that explicit memory declines in late adulthood to a greater degree than does implicit memory.

Process view. Process views focus on the nature of the cognitive processes that mediate performance on implicit and explicit memory tests. A number of different process views have been proposed. One variation that is widely used to explain age changes in memory is based on the distinction between integrative and elaborative processing (Mandler, 1980). Integrative processing occurs when the components of a stimulus are related to each other to form a perceptual Gestalt or unified whole. It is assumed that, once integrated, a target's memory representation has the tendency to become reintegrated even when only some of its components are presented as test cues (Graf \& Mandler, 1984; Graf, Mandler, \& Haden, 1982). Elaborative processing occurs when a target item is related to other items, to situational cues, and to prior knowledge. It is further assumed that by virtue of their different cues and instructions, performance on implicit memory tests is sensitive primarily to integrative processing during the study trial, whereas explicit remembering is facilitated primarily by elaborative processing during the study trial.

By this view, the finding that aging has a minimal or no detrimental effect on performance of implicit memory tests can be explained by suggesting that integrative processing occurs automatically, at least when familiar items such as words are used. In contrast, it is argued that explicit remembering is impaired because elaboration involves attention-demanding, controlled processes, and aging reduces the availability of the attentional resources required for this type of processing. We could extend this view to the developmental data and argue that implicit memory develops earlier than explicit memory because development increases the pool of attentional resources that are required only for the latter type of processing.

An alternative view that does not appeal to changes in attentional resources is based on the assumption that performance on any memory task involves the coordinated application of several component processes and that what changes over the course of life is subjects' ability to initiate and coordinate these components. By this view, implicit memory performance remains constant across the life span because integrative processing is self-coordinating-it is mediated by sensory and perceptual processes that are initiated and guided by the cues and instructions provided for testing. In contrast, explicit remembering varies across the life span because of subjects' changing ability to initiate and coordinate the components involved in elaboration. What is important to note is that this change in coordination reflects different factors in children and older adults: It is assumed that young preschoolers haven't yet developed the capacity for conceiving a mental model that includes and guides the components involved in elaboration; in contrast, older adults are capable of conceiving such models, but they may have difficulty executing them because the individual components do no longer flow as quickly and/or as fluently as they once did. By this interpretation, memory changes in early childhood and old age are viewed differently - as reflecting limits on competence and performance, respectively.

Task analysis views. Task analysis views focus on the requirements of different memory tests. An insightful model of this type was suggested by Craik (1983). He proposed that all memory tests can be arranged on a continuum that reflects the extent to which performance depends on subject-initiated activities, on one end, and the extent to which it is supported or guided by the test environment, on the other end. If we allow that the amount of environmental support is defined by the cues together with the instructions that are given to subjects, implicit memory tests are clearly clustered at one end of Craik's continuum (they provide a lot of environmental support), whereas explicit memory tests tend to be closer to the other end-the subject-initiated activities end. Craik proposed that older adults show normal performance on tests that provide a lot of environmental support and impaired performance on tests that require more subject-initiated activities. The reason for this pattern of effects, he argued, is that aging reduces the attentional capacity required for initiating and coordinating complex cognitive activities.

We can extend this view to memory development in early childhood. Young children show normal implicit memory because such tests provide enough constraints to initiate and direct retrieval. Explicit memory tests do not provide sufficient support, but development brings about an increase in the attentional capacity that is needed for the internal or mental coordination of the processes required for explicit remembering.

As with the process view discussed previously, I prefer an alternative to a capacity interpretation, which focuses on life-span changes in subjects' ability to initiate and coordinate memory processes. By this view, young children show impaired performance on tasks that provide little or no environmental support because they haven't yet developed the ability to conceive a mental model that includes and can guide the processes required for explicit remembering, whereas older adults can conceive such models but they may have difficulty executing them.

This general proposal is closely related to the position of Istomina (1975), who argued that during infancy and the preschool period, "memory processes [encoding, retrieval] are not independent processes, but are integrated into some other activity and are involuntary. Later, ... these processes are transformed into specific, internal acts" (p. 7). Istomina suggested that encoding and retrieval are processes that are initially embedded in other activities, such as playing games, and their use is initiated and guided by the content and structure of these activi- 
ties. This is exactly how we test implicit memory in children; we invite them to play a familiar game, and the structure of this game is the environmental support that initiates and guides retrieval. By Istomina's view, true remembering is made possible by the development of a new capacity for formulating conscious plans or mental models for initiating and guiding the processes required for intentional recollection (see Greenbaum \& Graf, 1989).

\section{REFERENCES}

BjorkLund, D. F., \& BjorkLuND, B. R. (1985). Organization versus item effects of an elaborated knowledge base on children's memory. Developmental Psychology, 21, 1120-1131.

Carroll, M., Byrne, B., \& KirSner, K. (1985). Autobiographical memory and perceptual learning: A developmental study using picture recognition, naming latency, and perceptual identification. Memory \& Cognition, 13, 273-279.

Craik, F. I. M. (1983). On the transfer of information from temporary to permanent memory. Philosophical Transactions of the Royal Society of London, Series B, 302, 341-359.

Graf, P., \& MANDler, G. (1984). Activation makes words more accessible but not necessarily more retrievable. Journal of Verbal Learning \& Verbal Behavior, 23, 553-568.

Graf, P., Mandler, G., \& Haden, P. E. (1982). Simulating amnesic symptoms in normal subjects. Science, 218, 1243-1244.

Graf, P., \& SChacter, D. L. (1985). Implicit and explicit memory for new associations in normal and amnesic subjects. Journal of Experimental Psychology: Learning, Memory, \& Cognition, 11, 501-518.

Greenbaum, J. L., \& Graf, P. (1989). Preschool period development of implicit and explicit remembering. Bulletin of the Psychonomic Society, 27, 417-420.
HowARD, D. V. (1986, November). The aging of implicit memory. Paper presented at the annual meeting of the Psychonomic Society, New Orleans.

HowARD, D. V. (1988). Implicit and explicit assessment of cognitive aging. In M. L. Howe \& E. J. Brainerd (Eds.), Cognitive development in adulthood: Progress in cognitive development research (pp. 337). New York: Springer-Verlag.

Howard, D. V., Heisey, J. G., \& Shaw, R. J. (1986). Aging and the priming of newly learned associations. Developmental Psychology, 22, 78-85.

Istomina, Z. M. (1975). The development of voluntary memory in preschool-children. Soviet Psychology, 12, 5-64.

KAUSLER, D. H. (1982). Experimental psychology and human aging. New York: Wiley.

Light, L. L., Singh, A. (1987). Implicit and explicit memory in young and older adults. Journal of Experimental Psychology: Learning, Memory, \& Cognition, 13, 531-541.

Light, L. L., Singh, A., CAPPS, J. L. (1986). The dissociation of memory and awareness in young and older adults. Journal of Clinical \& Experimental Neuropsychology, 8, 62-74.

MANDLER, G. (1980). Recognizing: The judgment of previous occurrence. Psychological Review, 87, 252-271.

PARKIN, A. J., \& STREete, S. (1988). Implicit and explicit memory in young children and adults. British Journal of Psychology, 79, 361-369.

Rabinowitz, J. C. (1986). Priming in episodic memory. Journal of Gerontology, 41, 204-213.

Rose, T. L., Yesavage, J. A., Hill, R. D., \& Bower, G. H. (1986). Priming effects and recognition memory in young and elderly adults. Experimental Aging Research, 12, 31-37.

Salthouse, T. A. (1982). Adult cognition. New York: Springer-Verlag.

SChaCTER, D. L., \& Moscovitch, M. (1984). Infants, amnesics, and dissociable memory systems. In M. Moscovitch (Ed.), Infant memory: Its relation to normal and pathological memory in humans and other animals (pp. 173-216). New York: Plenum. 